\title{
INTRODUCCIÓN EN LA DOCENCIA DEL CONCEPTO DESARROLLO SUSTENTABLE EN LA UNIVERSIDAD DE MOA
}

\author{
Juan Manuel Montero Peña ${ }^{1}$, \\ Hernando Hernández Pérez ${ }^{2}$, André Afonso Bambi ${ }^{3}$
}

\section{RESUMEN}

La introducción de los presupuestos teóricos del desarrollo sustentable en la sociedad se ha convertido en una necesidad social de prácticamente todas las actividades económicas, sociales y políticas del mundo. La educación superior es una de las vías a través de las cuales se puede lograr que la filosofía de la sustentabilidad se convierta en herramientas reales de la transformación de la realidad planetaria y el desarrollo sustentable sea un paradigma en este proceso, principalmente a través de la formación de profesionales. En esta investigación se realiza una propuesta de como introducir los presupuestos de este concepto en la Universidad de Moa. Se considera la necesidad de introducir los fundamentos del concepto en las asignaturas donde se estudia la relación hombre - naturaleza - sociedad por considerar que desde aquí se pueden crear las bases para comprender las complejidades epistemológicas del concepto desarrollo sustentable.

Palabras clave: Desarrollo sustentable. Educación superior. Transformación de la realidad.

\section{Como citar este documento - ABNT}

MONTERO PEÑA, Juan Manuel; HERNÁNDEZ PÉREZ, Hernando; BAMBI, André Afonso. Introducción en la docencia del concepto Desarrollo Sustentable en la Universidad de MOA. Revista Docência do Ensino Superior, Belo Horizonte, v. 10, e014969, p. 1-19, 2020. DOI: https://doi.org/10.35699/2237-5864.2020.14969.

Recebido em: 28/08/2019 Aprovado em: 26/11/2019 Publicado em: 09/04/2020

\footnotetext{
${ }^{1}$ Universidad de Moa “Dr. Antonio Núñez Jiménez", Cuba. ORCID: https://orcid.org/0000-0002-0869-1171. E-mail: jmpena@ismm.edu.cu, jmmonteropena@gmail.com. 2 Universidad de Moa "Dr. Antonio Núñez Jiménez", Cuba. ORCID: https://orcid.org/0000-0002-2507-4300. E-mail: hhernandez@ismm.edu.cu.

${ }^{3}$ Instituto Superior de Ciencias de Educación de Uige, Angola.

ORCID: http://orcid.org/0000-0003-2786-2041. E-mail: andrebambi2@gmail.com.
} 
INTRODUÇÃO NO ENSINO DO CONCEITO DE DESENVOLVIMENTO SUSTENTÁVEL NA UNIVERSIDADE DE MOA

\section{RESUMO}

A introdução dos pressupostos teóricos do desenvolvimento sustentável na sociedade tornou-se uma necessidade social de praticamente todas as atividades econômicas, sociais e políticas do mundo. O ensino superior é uma das maneiras pelas quais a filosofia da sustentabilidade pode se tornar uma ferramenta real para a transformação da realidade planetária, e o desenvolvimento sustentável é um paradigma nesse processo, principalmente por meio da formação profissional. Nesta pesquisa, é feita uma proposta sobre como introduzir os pressupostos desse conceito na Universidade de Moa. É necessário introduzir os fundamentos do conceito nos assuntos em que se estuda a relação homem natureza - sociedade, considerando que a partir daqui é possível criar a base para a compreensão das complexidades epistemológicas do conceito de desenvolvimento sustentável.

Palavras-chave: Desenvolvimento sustentável. Educação superior. Transformação da realidade.

\section{INTRODUCTION TO THE TEACHING CONCEPT OF SUSTAINABLE DEVELOPMENT AT THE UNIVERSITY OF MOA}

\section{ABSTRACT}

The introduction of theoretical sustainable development assumptions into society has become a social necessity for virtually all economic, social and political activities in the world. Higher education is one of the ways in which the sustainability philosophy can become a real tool for the transformation of the planetary reality and the sustainable development is a paradigm in this process, especially through professional formation. In this research, a proposal is made on how to introduce the assumptions of that concept at the University of Moa. It is necessary to introduce the fundamentals of the concept in the subjects in which man - nature - society relationship is studied, considering that from here it is possible to create the basis for understanding the epistemological complexities of the concept of sustainable development.

Keywords: Sustainable development. Higher education. Transformation of reality. 


\section{INTRODUÇÃO}

La presente investigación tiene como antecedentes la defensa de la Tesis de Doctorado de su autor principal en el año 2006, titulada "El desarrollo compensado como alternativa de sustentabilidad en la minería (aprehensión ético - cultural)" (MONTERO PEÑA, 2006), la Tesis de Maestría en el año 2001, titulada "El desarrollo sustentable en la minería" y el inicio de la "Maestría en desarrollo sustentable en la actividad minero - metalúrgica" (MONTERO PEÑA, 2014) el 30 de enero del año 2012.

En ambas tesis se defiende la necesidad de reconceptualización del concepto desarrollo sustentable por considerarlo una elaboración teórica totalmente descontextualizada. Sin embargo, este es un concepto necesario para el logro de un escenario mundial favorable para la conservación y protección de la naturaleza y para la elevación de la calidad de vida de la población.

De ahí la importancia de buscar vías para operacionalizar el concepto desarrollo sustentable en la vida socioeconómica, introducirlo en la práctica cotidiana para que se convierta en una herramienta real de transformación de la realidad. Una de las formas de lograrlo, es introduciendo sus presupuestos teóricos en la formación de profesionales universitarios.

Esta investigación tiene referentes relevantes que demuestran la pertinencia social de la misma. La Tesis de Maestría, titulada "Incorporación de las dimensiones de sustentabilidad en el currículo de Tecnología en Recursos Naturales renovables del IUTY", de la M. Sc. Marlin Montero Castillo, del Instituto Universitario de Tecnología de Yaracuy, en la República Bolivariana de Venezuela. Aquí se elabora un sistema de tareas docentes para la incorporación de las dimensiones de la sustentabilidad en los contenidos programáticos de la unidad curricular Ecología en la carrera de Tecnología en Recursos Naturales Renovables del IUTY ${ }^{4}$.

Un antecedente directamente relacionado con el tema es la defensa en febrero del año 2016 de las tesis de Maestría de los licenciados Frank Tejas Paz y Yaniset Fuentes Londres en los marcos de la "Maestría en desarrollo sustentable en la actividad minero - metalúrgica". Las tesis se realizaron en las carreras de Ingeniería en Minería e Ingeniería en Geología con el título de "Sistema de tareas docentes para implementar las dimensiones del desarrollo sustentable en la carrera de Ingeniería en Geología en el ISMMM de Moa "Dr. Antonio Núñez Jiménez" e indistintamente en Ingeniería en Minería. Estos resultados aún no se han introducido en ambas carreras.

\footnotetext{
${ }^{4}$ Montero Peña, J. M. (2014): "Incorporación de las dimensiones de sustentabilidad en el currículo de Tecnología en Recursos Naturales renovables del Instituto Universitario de Tecnología de Yaracuy (IUTY)". Lara. Tesis presentada en opción al Grado Académico de Máster en Ciencia, tecnología y sociedad. Facultad de Humanidades. Universidad de Cienfuegos.
} 
Actualmente está en curso otra investigación sobre el tema de un maestrando de la "Maestría en desarrollo sustentable en la actividad minero - metalúrgica", se trata de Hélio Mbige Miguel Dos Santos quien está investigando la introducción del concepto desarrollo sustentable en la carrera de Ingeniería en Minería. Todas estas investigaciones, referidas con anterioridad, son tutoradas por el autor principal de este artículo.

En este artículo se realiza una propuesta de introducción de las bases conceptuales del concepto desarrollo sustentable en las asignaturas de corte filosófico, económico y sociopolítico en la Universidad de Moa "Dr. Antonio Núñez Jiménez", todo ello considerando que las asignaturas de esta deben crear las habilidades para articular en los currículos de todas las carreras los presupuestos teóricos de este modelo de desarrollo.

Este proceso debe comenzar por la introducción de los presupuestos teóricos del desarrollo sustentable a través de la utilización de las categorías de las dimensiones de la sustentabilidad como ejes transversales en cada una de las carreras que se estudian en la Universidad de Moa “Dr. Antonio Núñez Jiménez".

\section{CONCEPTO DESARROLLO SUSTENTABLE Y EDUCACIÓN SUPERIOR}

El concepto tiene sus momentos de sacralización en el Informe de la Comisión Brundtland, de 1987, también conocido como "Nuestro Futuro Común", y en los documentos surgidos de la Cumbre de la Tierra, en Río de Janeiro, en 1992. Sin embargo, aún no existe un acuerdo académico sobre qué entender por desarrollo sustentable. Este es un tema de extraordinaria importancia a nivel mundial, tanto que las Organización de las Naciones Unidas (ONU) a través de la Resolución 57/254 de la Asamblea General de las Naciones Unidas, declara el periodo 2005-2014 como una década dedicada a fortalecer la educación y reorientarla hacia los objetivos del desarrollo sustentable. Obviamente este reto tuvo su mayor impacto en las universidades.

Sobre la relación del concepto con la educación superior existen dos hitos importantes que se deben tener en cuenta, se trata de la firma por rectores de todo el mundo en 1991 la Declaración de Talloiers para un futuro sostenible, a partir de la cual se creó la "Asociation of University Leaders for sustainable future", de la misma forma que en 1993 la Conferencia de rectores de Europa dio a luz a la Declaración de Universidades por un desarrollo sustentable, que daría origen a la "Asociación Copernicus". Es evidente la intención de la universidad de convertirse en un actor estratégico de la sociedad para el desarrollo sustentable.

Este objetivo, por lo general, se ha dirigido hacia la introducción de la educación ambiental en la enseñanza universitaria, sin considerar la necesidad de introducir directamente el concepto desarrollo sustentable, sus dimensiones, categorías y principios. 
El nuevo siglo le está imponiendo retos importantes a la educación que deben ser asumidos por las universidades de manera responsable, en tal sentido se asume aquí como válida la afirmación de Díaz y Escárcega (2009) cuando aseguran que:

La educación del siglo XXI no sólo debe responder a la necesidad de proveer y mantener el actual sistema económico, sino que también cumpla con la misión humanista de permitir a cada quien realizar plenamente sus talentos y aptitudes en la búsqueda de un proceso respetuoso del ambiente humano, natural y cultural y que haga posible cimentar las bases del desarrollo sustentable en distintas escalas (DÍAZ; ESCÁRCEGA, 2009, p.225).

Esta es una idea básica cuando se trata de la necesidad de poseer sistemas educativos que formen ciudadanos capaces de desarrollar su talento con valores que les permitan respetar el medio ambiente humano, natural y cultural, para lo cual es necesario formarlo con programas que privilegien esos valores, todos presentes teóricamente en la filosofía del desarrollo sustentable. Pero este proceso no es tan sencillo, en un mundo donde se ha impuesto la racionalidad instrumental de la modernidad, a decir de Enrique Leff (1998).

La complejidad creciente y la agudización de los problemas socioambientales, generados por el triunfo de la racionalidad económica y de la razón tecnológica, han llevado a plantear la necesidad de reorientar los procesos de producción y aplicación de conocimientos, así como la formación de habilidades profesionales, para conducir un proceso de transición hacia el desarrollo sustentable (LEFF, 1998, p.225).

Como se puede ver los autores citados están hablando de la necesidad de formar habilidades para transitar hacia una sociedad sustentable. La respuesta a esta problemática es muy compleja, pasa por diferentes visiones que aportan mucho a la elaboración de una propuesta para abordarla. Para Eduardo Gudynas:

Uno de los requisitos para la comprensión ecológica de la tierra es el conocimiento de la ecología, pero esto no está incluido de ningún modo en la 'educación', de hecho, gran parte de la educación superior parece eludir deliberadamente los conceptos ecológicos. El conocimiento de la ecología no siempre se obtiene en los cursos que ostentan un título ecológico, pues es igualmente probable que lleve las etiquetas de geografía, botánica, agronomía, historia o economía. Esto no nos debe extrañar, pero cualquiera que sea la etiqueta, la educación ecológica es escasa (GUDYNAS, 2004, p.204-205).

Esta idea es muy importante porque lleva a la introducción del análisis holístico en los programas, la solución del necesario contenido ecológico en la enseñanza universitaria no se resolverá con una asignatura directamente dedicada a la Ecología. El problema es mucho más complejo, se trata de asumir los contenidos curriculares de cualquier carrera 
universitaria con un enfoque ecológico lo cual es mucho más complejo y requiere de cambios al interior de las disciplinas.

En esta misma línea de pensamiento Manuel Villarruel Fuentes considera:

[...] la urgencia de transitar de un paradigma racional que privilegió durante décadas el control y uso eficiente de la naturaleza, hacia otro más congruente con el sentido de supervivencia y continuidad de la especie humana, obliga a replantear el papel educativo de los institutos y universidades, repensando la educación superior desde los planos conceptuales, metodológicos y de valores, buscando alcanzar una verdadera transformación cultural, que se signifique por trascender el habitual discurso ecologista oficial (VILLARRUEL FUENTES, 2006, p.1).

La idea de los cambios en los programas es inevitable, no se puede continuar defendiendo la visión de que una asignatura con contenidos directamente "ambientales" resolverá el problema del imprescindible enfoque hacia lo ambiental que no acaba de asentarse en las universidades. Es apreciable la necesidad de superar los estancos disciplinares que la modernidad impuso en los diseños curriculares.

\section{EL TRATAMIENTO DEL PROBLEMA DEL DESARROLLO SUSTENTABLE EN CUBA}

Este es un problema de tanta complejidad que necesita ser abordado desde la mirada de varias ciencias. Los procesos vinculados con la relación hombre - naturaleza - sociedad hay que investigarlos desde la multi y la transdisciplinariedad, de no hacerlo así se corre el riesgo de no poder englobar la complejidad de las relaciones de un fenómeno que abarca prácticamente todos los campos de la actividad humana.

En Cuba existen, en todas las carreras universitarias, disciplinas que crean las bases para el analice de los problemas del medio ambiente, pero en ellas apenas se aborda el problema de la sustentabilidad. Se trata de las asignaturas de corte socio - humanista.

En las carreras que se estudian en la Universidad de Moa "Dr. Antonio Núñez Jiménez" a pesar de ser un centro donde se cursan, como carreras rectoras, las llamadas ciencias de la tierra, en ninguna existe una disciplina o asignatura dedicada a la sustentabilidad, independientemente de que el problema ambiental es tratado en varias disciplinas. Esta constituye, indudablemente una limitación de los programas de estudios.

El Plan de estudios D de la carrera Ingeniería de Minas plantea como problema fundamental "La necesidad de aprovechar los recursos minerales y funcionales de la corteza terrestre con un enfoque sostenible". ${ }^{5}$ Lo sostenible aparece en este plan en 5 ocasiones, en la página 5

\footnotetext{
${ }^{5}$ Plan de Estudios E. Carrera de Ingeniería de Minas, Ministerio de Educación Superior. Moa, 2017. p. 5
} 
Hernando Hernández Pérez, André Afonso Bambi

en el problema fundamental de la carrera, en la página 6 cuando se mencionan las funciones del profesional, en la página 11 , en dos ocasiones, en la fundamentación de la disciplina Marxismo - leninismo, en la página 13 cuando se menciona la necesidad de la actualización jurídica del "socialismo próspero y sostenible" que se edifica en Cuba, en la página 24 en la fundamentación de la disciplina Historia de Cuba y en el mismo sentido anterior mencionando la frase "socialismo próspero y sostenible", en la fundamentación de la disciplina Ergonomía Ambiental, en una de las habilidades que debe crear esta disciplina y en el título de una bibliografía, la número 20, de la disciplina Geomecánica.

Como "desarrollo sostenible" se menciona en tres ocasiones, en la citada literatura de la disciplina Geomecánica, en la página 51, en la fundamentación de la disciplina Ergonomía Ambiental, en la página 59 y en las funciones del profesional en la página 6 . Sin embargo, en los problemas derivados del problema principal y en el modo de actuación del profesional, solo se menciona el concepto desarrollo sustentable en la asignatura Problemas Sociales de la Ciencia y la Tecnología.

En la carrera Metalurgia y Materiales la situación es prácticamente similar, se utiliza el término en 19 ocasiones, en la página 8 en la definición del problema de la carrera, en la página 10 en el objetivo 1 de la carrera relacionado con formar profesionales capaces de contribuir al desarrollo sostenible, en las páginas 24 y 25 en la fundamentación de la disciplina Marxismo - leninismo, en la 25 dentro de los objetivos de esta disciplina, sin embargo no aparece dentro de los contenidos de ningunas de sus asignaturas.

Nuevamente aparece en la página 26, como declaración en la actualización jurídica del modelo, dentro de los conocimientos a adquirir en la disciplina Marxismo - leninismo y en la página 28 entre los conocimientos de la disciplina Historia de Cuba. En la disciplina Preparación para la defensa se declara, pero no se operacionaliza.

La aparición del término en la disciplina Educación Física, dentro de sus objetivos, en la página 50 demuestra se declara el término, así aparece en los objetivos de la disciplina Matemática en la página 56, en los de Física en la página 62, en los de Química en la página 69, en los de Mecánica en la página 75, en los de Fundamentos de Automatización en la página 79 y 80, en los de Gestión Empresarial, en los de Ciencia e Ingeniería de los Materiales en la página 89, en los de Procesos y Operaciones Unitarias en la página 96 y finalmente, en la página 103 en la disciplina Tecnología Metalúrgicas y de materiales. ${ }^{6}$

La situación que se presenta en el Plan de Estudio e de la carrera Metalurgia y Materiales es muy interesante, se reconoce en los objetivos de 13 disciplinas la necesidad del logro de un desarrollo sostenible, pero no se indica en los conocimientos a adquirir como se logrará.

\footnotetext{
${ }^{6}$ Plan de Estudio E. Carrera de Metalurgia y Materiales, Ministerio de Educación Superior. Moa, 2018.
} 
Hernando Hernández Pérez, André Afonso Bambi

La propuesta que se presenta en esta investigación parte de analizar el significado de este concepto para la ciencia, especialmente se trata de encontrar la forma de integrar los presupuestos teóricos de diferentes disciplinas para construir un conocimiento que integre las relaciones derivadas de la "satisfacción de las necesidades de las generaciones actuales, sin comprometer la capacidad de las generaciones futuras de satisfacer las suyas".

En un segundo momento se analizará como fundamentar científicamente las dimensiones del desarrollo sustentable y su introducción en la docencia de pre y postgrado. Por eso es imprescindible determinar de qué manera operacionalizar estas dimensiones en los planes de estudios de las carreras universitarias, en los proyectos económicos y en las actividades de las organizaciones políticas, sociales y gubernamentales del país.

En un tercer momento se realizará una propuesta del significado metodológico del concepto desarrollo sustentable en la elaboración de una estrategia para introducirlo en la docencia, para lo cual se proponen acciones estratégicas, entre ellas las dirigidas a las instituciones de la sociedad, que incluyen un sistema de superación desde el pregrado y todo el sistema de superación profesional de la Universidad de Moa "Dr. Antonio Núñez Jiménez" en esta temática.

\section{EL CONCEPTO DESARROLLO SUSTENTABLE: CIENCIAS RELACIONADAS CON EL PROBLEMA}

En la numerosa literatura consultada se pueden encontrar diferentes miradas al concepto, todas a partir de la elaboración conceptual planteada en el Informe de la Comisión Brundtland en 1987. Para la gran mayoría de los autores consultados, el desarrollo sustentable es aquel tipo especial de desarrollo, entendido no como estado de estática armonía, sino como un proceso de cambio en el cual la explotación de los recursos, la dirección de las inversiones, el desarrollo tecnológico y los cambios institucionales están dirigidos a garantizar la satisfacción de las necesidades de las generaciones actuales, sin comprometer la capacidad de las futuras generaciones de satisfacer las suyas.

Es muy difícil determinar cuáles serían las disciplinas y asignaturas que se relacionan con la explicación de qué entender por desarrollo sustentable y la introducción de este concepto en la enseñanza universitaria. Ante todo, como se expresa con anterioridad, este en un problema que hay que estudiarlo desde la multi y la transdisciplinariedad pero existen ciencias particulares que le ofrecen al alumno herramientas para comprender, durante toda la carrera, como operacionalizar este concepto. Es decir; hay que buscar lo multidisciplinar y lo transdisciplinar, pero esto no significa que se deba eliminar la especialidad.

El problema es llevarle al alumno una visión global, holística del tema para que pueda comprender que los conceptos, leyes y categorías del desarrollo sustentable se encuentran 
integrado en un universo más global, en estructuras metodológicas que son compartidas por varias disciplinas. Por eso se hace imprescindible elaborar un sistema de superación que prepare a los docentes para explicar de esta forma qué es la sustentabilidad, esta es una acción a más largo plazo.

En los planes de estudios de las carreras de la Universidad de Moa "Dr. Antonio Núñez Jiménez" esta perspectiva no existe, hay que introducirla y para ello se hace imprescindible formar a los profesores. Si se revisa el plan de estudios de cualquier carrera no se encontrará ninguna explicación a la relación que existe entre la satisfacción de las necesidades de las generaciones actuales, la actividad minera, la composición química de la naturaleza y la explicación que las ciencias sociales, económicas y humanísticas ofrecen sobre la producción de bienes materiales. Esa visión en la explicación de la sustentabilidad no existe.

Por eso es imprescindible tener en cuenta algunas ideas que pueden ser esenciales en la explicación de cómo convertir el desarrollo sustentable en un eje articulador para la explicación de la relación hombre - naturaleza - sociedad en la universidad. Para hacer esta propuesta se tomará como referencia las carreras de Minería, Metalurgia y Materiales y Geología.

- En primer lugar; definir los conceptos, leyes y categorías fundamentales del concepto desarrollo sustentable que necesitan introducirse en cada carrera.

- En segundo lugar; determinar cómo se introducirán los conceptos, leyes y categorías que se decidan en cada asignatura. Por ejemplo, como se trabajará el concepto necesidades en diferentes asignaturas para que el estudiante comprenda su generalidad.

- En tercer lugar; definir de acuerdo con los intereses de cada carrera si es necesario una disciplina integradora para evaluar la introducción de la teoría de la sustentabilidad en la docencia.

- En cuarto lugar; evaluar en el trabajo de diploma de cada estudiante como se introducen las dimensiones del desarrollo sustentable como variables obligatorias para la defensa del trabajo.

- En quinto lugar; evaluar en las prácticas laborales de cada año como se pueden operacionalizar las dimensiones de la sustentabilidad.

En sentido general se debe definir, con claridad, que no se trata de seleccionar un grupo de asignaturas para introducir el concepto en la docencia, se trata como se ha planteado aquí 
Hernando Hernández Pérez, André Afonso Bambi

de convertir la teoría de la sustentabilidad en una nueva forma de indagar, concebir y planificar la relación del hombre con la naturaleza y con otros hombres.

\section{LA OPERACIONALIZACIÓN DE LAS DIMENSIONES DE LA SUSTENTABILIDAD EN LA DOCENCIA}

La introducción de esta problemática en la docencia posee algunos presupuestos que es muy importante que se valoren, antes de comenzar a analizar la problemática de las dimensiones. El pregrado ocupa un espacio vital en la formación de valores ambientales en la misma medida que de una u otra forma en los diferentes modelos de profesionales de las universidades cubanas están incluidas las invariantes ecológicas en las disciplinas de las carreras, tanto en la formación de ingenieros como en las humanidades.

La formación de ingenieros exige de una cultura tecnológica en la cual interactúan dialécticamente elementos técnicos, organizacionales y culturales, y eso es precisamente la esencia misma del desarrollo sustentable: ver la tecnología como un sistema cultural resultado lógico del desarrollo de la humanidad donde intervienen los conocimientos, los hábitos, las necesidades y las valoraciones de cada época histórica. A partir de esta concepción se debe llevar a los estudiantes la idea de la tecnología como un instrumento consciente de transformación de la realidad estrechamente ligado a grupos sociales específicos. En este proceso la educación posee una responsabilidad suprema al estar obligada a encontrar fórmulas para lograr formar desde esta perspectiva valores ambientales propios del proyecto social cubano, sin perder la dialéctica de la relación individuo-sociedad como un momento concreto de la realización individual del sujeto, es decir no se puede desconocer que el hombre es una individualidad con intereses singulares.

Este es un tema que ha abordado el autor principal de este artículo en varias ocasiones, pero nunca lo ha hecho con fines pedagógicos. Sin embargo; tiene la absoluta convicción que la única vía de alcanzar el desarrollo sustentable en cualquier país es logrando introducir las dimensiones de la sustentabilidad en la enseñanza, especialmente en la docencia universitaria a través de los currículos de las carreras, de proyectos universitarios, en las prácticas laborales y en las estrategias particulares.

Roberto P. Guimarães en un artículo emblemático sobre el tema, "El desarrollo sustentable:) propuesta alternativa o retórica neoliberal?" de 1994, planteaba,

La sustentabilidad ecológica, - según este autor - se refiere a la base física del proceso de crecimiento y promueve la necesidad de mantener un stock de recursos naturales incorporados a las actividades productivas. La sustentabilidad en el caso de los recursos naturales renovables, existe si la tasa de utilización es equivalente a la tasa de recomposición del recurso en los procesos naturales que tienen lugar en la naturaleza. En el caso de los 
recursos naturales no renovables, la tasa de utilización debe ser equivalente a la tasa de sustitución del recurso en el proceso productivo por el período de tiempo previsto para su agotamiento (medido por las reservas naturales y la tasa de utilización) (GUIMARÃES, 1994, p.51).

Para el caso de los recursos no renovables, como es la minería, es imprescindible que el egresado una vez en la producción pueda trabajar por contribuir a comprender la necesidad de una economía que planifique como tendrá lugar la relación explotación del recurso planificación de nuevas actividades económicas para sustituir las que desaparecerán con el agotamiento del recurso natural. Como se puede valorar es imposible lograr la comprensión de este problema si no se hace utilizando los contenidos de los campos disciplinares de más de una asignatura, de las que explican cómo se produce la explotación de los recursos naturales y de otras para explicar el funcionamiento de la sociedad.

Pero este problema es mucho más complejo cuando entra a valorar aspectos que tienen una incidencia directa en la consecución de políticas de desarrollo como es el caso de las inversiones. Tanto en el caso de los recursos no - renovables como los renovables la sustentabilidad se garantiza en las inversiones, en este proceso hay que planificar los ritmos de explotación, las emisiones de desechos, la superación de los recursos humanos, la reconversión industrial, el cambio tecnológico y toda la política para la explotación sustentable de los recursos.

Para el autor citado con anterioridad:

la sustentabilidad ambiental dice relación con la mantención de la capacidad de sustento de los ecosistemas [...]. En primer lugar, las tasas de emisión de desechos como resultado de la actividad económica deben equivaler a las tasas de regeneración, las cuales son determinadas por la capacidad de recuperación del ecosistema [...]. Un segundo criterio de sustentabilidad ambiental, sería la reconversión industrial con énfasis en la reducción de la entropía, es decir, privilegiando la conservación de la energía y las fuentes renovables. Lo anterior significa que tanto las "tasas de recomposición" (para los recursos naturales) como la "tasa de regeneración" (para los ecosistemas) deben ser tratadas como "capital natural". La incapacidad de manutención de estas tasas debe ser tratada, por tanto, como consumo de capital, o sea, no sustentable (GUIMARÃES, 1994, p.51).

El análisis de los planes de estudios de las carreras mencionadas demostró que existen disciplinas como la Física, la Química, la Geoquímica, la Geofísica o la Geomecánica entre otras donde se introducen contenidos que permiten al estudiante entender esta dimensión, pero aún no es suficiente. No existe un tratamiento metodológico dirigido a ver la vinculación de estos contenidos con el desarrollo sustentable. Esto hay que enseñarlo, hay 
que formar esas habilidades para que el egresado en su actividad laboral pueda aplicarlas en la búsqueda de la sustentabilidad de las actividades en las que labora.

La sustentabilidad social, por su parte,

tiene como objeto el mejoramiento de la calidad de vida de la población. Para el caso específico de los países del Sur, con graves problemas de exclusión social, los criterios básicos debieran ser los de justicia distributiva, para el caso de la distribución de bienes y de servicios y de la universalización de la cobertura, para las políticas globales de educación, salud, vivienda y seguridad social (GUIMARÃES, 1994, p.52).

Esta mirada al problema de la sustentabilidad no existe en los planes de estudios porque las disciplinas de tipo sociohumanista y con perfiles afines para la explicación de esta problemática se quedan en la generalidad y no va a la explicación de los problemas del crecimiento y el desarrollo lo cual permitiría entender el desarrollo sustentable desde la organicidad de sus dimensiones. Esta es la esencia misma del desarrollo sustentable, las competencias de esta dimensión hay que formarlas para introducirlas en algún momento del proceso docente, de acuerdo a las características de cada carrera.

Para Guimarães la sustentabilidad política:

[...] se encuentra estrechamente vinculada al proceso de construcción de la ciudadanía, y busca garantizar la incorporación plena de las personas al proceso de desarrollo. Ésta se resume a nivel micro, a la democratización de la sociedad, y a nivel macro, a la democratización del estado. El primer objetivo supone el fortalecimiento de las organizaciones sociales y comunitarias, la redistribución de los recursos y de la información hacia los sectores subordinados, el incremento de la capacidad de análisis de sus organizaciones, y la capacitación para la toma de decisiones; mientras el segundo se logra a través de la apertura del aparato estatal al control ciudadano, la reactualización de los partidos políticos y de los procesos electorales, y por la incorporación del concepto de responsabilidad en la actividad pública (GUIMARÃES, 1994, p.53).

Evidentemente esta es una dimensión que expresa, de forma orgánica, el espíritu de la sustentabilidad. De ahí la necesidad de formar las competencias para un egresado que contribuya en estos procesos y de una universidad que genere sinergias positivas hacia un tipo de desarrollo que está en construcción. Hasta el momento, con estos planes de estudios, no se logra la formación de una cultura de la sustentabilidad.

Definitivamente queda mucho trabajo por hacer en este campo, es imprescindible comenzar a trabajar de inmediato en una propuesta integradora en la Universidad de Moa "Dr. Antonio Núñez Jiménez" dirigida a introducir de forma holística el concepto desarrollo sustentable en la docencia, para ello se proponen algunas acciones en este trabajo. 


\section{ACCIONES PARA INTRODUCIR EL DESARROLLO SUSTENTABLE EN EL CURRÍCULO} UNIVERSITARIO DE LAS CARRERAS DE LAS CIENCIAS DE LA TIERRA EN LA UNIVERSIDAD DE MOA

Esta investigación posee un primer producto que se propone desarrollar acciones para cumplir con este lineamiento, se trata de la "Maestría en desarrollo sustentable en la actividad minero - metalúrgica". Las acciones propuestas entran dentro de la "Estrategia Maestra para la promoción e implementación de un enfoque de desarrollo sustentable en las instituciones de la educación superior y en la sociedad".

1. Introducir en la docencia de pregrado el concepto, las leyes, categorías y dimensiones del desarrollo sustentable, a partir de una enseñanza inter, multi y transdisciplinaria que muestre al estudiante que la comprensión de los problemas de la sustentabilidad del desarrollo económico se logrará en la integración de saberes.

2. Desarrollar acciones, en el pregrado, que garanticen la introducción del enfoque de desarrollo sustentable en los trabajos de diplomas, como una vía de garantizar su pertinencia social, como una primera forma de operacionalizar las dimensiones de la sustentabilidad en la práctica social.

3. Promover y controlar la institucionalización del tratamiento del desarrollo sustentable en los proyectos de investigación universitarios, en los centros de investigación con los que se desarrollan acciones de I + D y en la proyección de todas las acciones dirigidas al desarrollo económico y social desde la universidad.

4. Garantizar que las variables relacionadas con el desarrollo sustentable se introduzcan en todas las modalidades de la docencia de postgrado, desarrollando un sistema que evalúe periódicamente como estas contribuyen al rigor académico de la docencia y a la pertinencia social de cada modalidad.

5. Elaborar Sistema de Superación sobre el desarrollo sustentable como una forma de superar a la comunidad universitaria en este tema y de generalizar en la sociedad el enfoque de la sustentabilidad como una nueva forma de encarar el crecimiento y el desarrollo.

6. Realizar una caracterización prospectiva del escenario universitario donde actúa la Universidad de Moa "Dr. Antonio Núñez Jiménez" en Cuba, en América latina y sus proyecciones hacia otras zonas geográficas como una forma de garantizar la sustentabilidad de los procesos desarrollados por la institución y su integración, expresados en un sistema de gestión orientado a la calidad. 
7. Instrumentar un sistema de evaluación continua, para conocer los resultados de la aplicación de la estrategia de sustentabilidad la Universidad de Moa "Dr. Antonio Núñez Jiménez", que permita introducir los cambios necesarios ante las posibles desviaciones que tengan lugar en su instrumentación.

En el pregrado estas acciones se pueden introducir en las asignaturas del ciclo básico de cada carrera, lógicamente, con las distinciones que el problema fundamental de cada una de ellas exige y el modo de actuación del profesional que egresará. En este primer trabajo se realiza una propuesta de la introducción del concepto desarrollo sustentable en las asignaturas de corte socio - humanista.

\section{ACCIONES A DESARROLLAR POR CADA UNA DE LAS ASIGNATURAS DE CORTE SOCIO- HUMANISTA}

\section{Asignatura Filosofía y Sociedad}

1. En la relación hombre - naturaleza - sociedad explicar la relación establecida por el hombre durante el proceso productivo, especialmente la forma en que el hombre utiliza los medios de producción. Especialmente es importante que se analice en el modo de producción la forma en la ciencia se convierte en una fuerza productiva directa.

2. En el modo de producción analizar como las relaciones de producción influyen sobre la posición que adopta el hombre en los diferentes mecanismos de producción de acuerdo a la formación económico social.

3. En el tema de los valores analizar el lugar que desempeña la sociedad en la formación de las necesidades de cada individuo, especialmente relacionando estas con la cultura y la identidad de cada grupo social.

4. Desarrollar un análisis crítico en el tema del sistema político los mecanismos de participación popular como elementos reguladores del funcionamiento de cada sociedad, especialmente de la formación de los órganos populares de control y de las organizaciones comunitarias.

5. Establecer la relación que existe entre las formas de la conciencia social y la actuación del individuo en cada escenario social donde desarrolla su vida, fundamentalmente como puede la conciencia social convertirse en un elemento mediador entre situaciones de vulnerabilidad que provocan amenazas y el medio ambiente. 
Asignatura Economía Política del Capitalismo

1. Explicar en el tema de la teoría valor - trabajo como adquieren valor los productos del trabajo humano en el proceso de producción. Principalmente se debe explicar que el valor de cualquier producto se adquiere a través del trabajo.

2. En el tema Categorías, leyes, tendencias y contradicciones esenciales del capitalismo explicar el lugar desempeñado por los recursos naturales, la ciencia y la revolución industrial como mediadores de la relación hombre - naturaleza - sociedad.

3. Analizar como el mercado es un elemento decisivo en la conformación de los estándares de la producción capitalista, es imprescindible que en este tema se analicen todos los factores de la producción capitalista.

4. En esta asignatura es básico que se utilice el tema de la relación desarrollo subdesarrollo para explicar las diferencias existentes el desarrollo de las naciones a nivel mundial y como la pobreza es causa y consecuencia de la depredación de la naturaleza y una de las barreras fundamentales para alcanzar el desarrollo sustentable.

5. Analizar en esta asignatura los hitos económicos que condujeron a la humanidad a la necesidad del planteamiento del modelo teórico del desarrollo sustentable. Es preciso que se analice los fundamentos económicos de este proceso.

\section{Asignatura Economía Política de la Construcción del Socialismo}

1. En el tema de la experiencia de la construcción socialista explicar la relación entre el socialismo y los fundamentos teóricos del desarrollo sustentable, primordialmente todo lo relacionado con las categorías equidad, justicia social y distribución equitativa.

2. Explicar en el tema de las relaciones de producción en la construcción del socialismo en condiciones de subdesarrollo como impacta el medio ambiente la producción en condiciones de marginalidad económica, utilizar como ejemplo las prácticas productivas no - sustentables en los países del bloque económico socialista.

3. En el tema del mecanismo económico de la construcción socialista valorar como entran los factores ambientales en la producción y como pueden protegerse para garantizar la continuidad de la producción. 
4. En la temática de la producción y reproducción del sistema de producción socialista explicar la forma en que se puede resolver el problema de la utilización de la fuerza laboral empleada en las empresas que operan con recursos naturales no renovables una vez que estos se agoten.

5. Valorar la relación existente entre los conceptos desarrollo humano y desarrollo sustentable y su relación con la lógica que presupone asumir el desarrollo sustentable como estilo de desarrollo en Cuba.

\section{Asignatura Teoría Sociopolítica}

1. En el tema de los grupos de presión y su redimensionamiento en la vida política de la sociedad valorar el papel de los grupos ambientalistas en la política y su impacto en la conformación de sociedades sustentables.

2. Los mecanismos de participación popular en la toma de decisiones ambientales, lugar de las organizaciones comunitarias, las instituciones y los ciudadanos en la conformación de un escenario favorable para el logro de una sociedad sustentable.

3. La globalización como factor de la toma de decisiones políticas, especialmente valorar todo lo relacionado con los mecanismos de cambio de la deuda externa por ecología y su incidencia en los países subdesarrollados.

4. Las organizaciones ambientales internacionales, su importancia en la conformación de escenarios mundiales de participación y concertación de acuerdos ambientales.

5. Los problemas ambientales en los programas políticos de los partidos tradicionales de las sociedades capitalistas y la continuidad de estos como partidos en el poder.

\section{Asignatura Problemas Sociales de la Ciencia y la Tecnología}

1. En el tema de la ciencia y la tecnología como procesos sociales analizar el impacto de la ciencia y la tecnología sobre el medio ambiente a partir de considera su carácter clasista.

2. Introducir en esta asignatura el tratamiento a los problemas de la relación medio ambiente - desarrollo que faciliten explicar el impacto de los factores ambientales en el desarrollo y el lugar de los factores sociopolíticos. 
3. En el tema de la transferencia de tecnología explicar la influencia de este proceso en los sistemas de producción locales, fundamentalmente en aquellos que basan sus resultados en el uso de técnicas tradicionales.

4. El tema de la responsabilidad moral de la intelectualidad científica debe llevar al análisis de la responsabilidad moral del científico, de los códigos de éticas profesionales y a la introducción del tema de la responsabilidad social empresarial como elementos claves para fomentar el desarrollo de la responsabilidad moral de los tomadores de decisiones ambientales.

5. Explicar la naturaleza social del cambio tecnológico y su importancia en los cambios de paradigmas en las diferentes olas de desarrollo tecnológico hasta llegar a la etapa actual, haciendo énfasis en el reconocimiento del papel de los flujos transfronterizo de datos y sus impactos en el nuevo escenario económico mundial.

\section{A MODO DE CONCLUSIONES}

La introducción del concepto desarrollo sustentable en la docencia precisa que las categorías de cada una de las dimensiones de la sustentabilidad se traten de forma sistémica en una o más disciplina de las carreras que se estudian en la Universidad de Moa "Dr. Antonio Núñez Jiménez".

El carácter multi y transdisciplinar de los procesos vinculados con el desarrollo sustentable exigen la superación de barreras relacionadas con la impartición de una docencia de holística en una sociedad diseñada para pensar de forma lineal, incapaz de comprender en toda su magnitud las relaciones complejas entre la universidad, la sociedad y la formación de valores. Ello demanda de profesores con una capacitación diferente a la actual.

La introducción de las bases teóricas del desarrollo sustentable en las asignaturas de corte socio - humanista es apenas el punto de partida para que los estudiantes comprendan la necesidad de introducir las categorías de las dimensiones de la sustentabilidad, sus principios y filosofía en general en el currículo universitario, no como una invariante, como una forma particular de indagar y transformar la realidad donde actuarán los egresados de la Universidad.

La introducción del concepto desarrollo sustentable en la docencia universitaria es la vía para garantizar que los profesionales se apropien de las herramientas que les faciliten introducir los fundamentos de este modelo en la práctica socioeconómica, pero no es una garantía de que lo harán en sus puestos laborales. De ahí la necesidad de elaborar instrumentos que permitan medir en las prácticas profesionales como se apropiaron de ese 
conocimiento, para ello hay que diseñar nuevas formas de concebir estos ejercicios que hoy no existen en la Universidad.

\section{REFERENCIAS BIBLIOGRÁFICAS}

DÍAZ, Reynol; ESCÁRCEGA, Susana. Desarrollo sustentable: Oportunidad para la vida. México: McGraw - Hill/Interamericana Ed., S. A. de CV, 2009.

LEFF, Enrique. Saber ambiental: Sustentabilidad, racionalidad, complejidad, poder. México: Siglo Veintiuno Ed., S. A de CV, 1998.

GUIMARÃES, Roberto P. El desarrollo sustentable: ¿Propuesta alternativa o retórica neoliberal?. Revista EURE, Santiago de Chile, v. 20, n. 61, p. 41-56, 1994.

GUDYNAS, Eduardo. Economía, Ecología y Ética del desarrollo sostenible. Uruguay: Editora Coscoroba, 2004.

MONTERO PEÑA, Juan Manuel. El desarrollo sustentable en la minería. 2001. Orientadores: Clara Elisa Miranda Vera, Eulicer Fernández Maresma. Tesis de Maestría (Tesis en Opcion al Titulo Academico de Master en Ciencia - Tecnologia - Sociedad) - Facultad de Humanidades, Universidad de Cienfuegos, Moa, 2001.

MONTERO PEÑA, Juan Manuel. El desarrollo compensado como alternativa de sustentabilidad en la minería (aprehensión ético - cultural). 2006. Orientadores: Jorge Núñez Jover, Eulicer Fernández Maresma, José Otaño Noguel. Tesis de Doctorado (Tesis en Opción al Grado Científico de Doctor en Ciencias Filosóficas) - Facultad de Filosofía, Universidad de la Habana, La Habana, 2006.

MONTERO PEÑA, Juan Manuel. La "Maestría en desarrollo sustentable en la actividad minero - metalúrgica": una visión desde los estudios sociales de la ciencia y la tecnología. Revista Caribeña de las Ciencias Sociales, Universidad de Málaga, España, ene. 2014. Disponible en: http://xn--caribea-9za.eumed.net/wp-content/uploads/estudios-sociales.pdf. Acceso en: 27 mayo 2019.

VILLARRUEL FUENTES, M. Educación Superior y desarrollo sustentable. Revista Iberoamericana de Educación. v. 38, n. 4, p. 1-7, 10 abr. 2006. DOI: https://doi.org/10.35362/rie3842641. Disponible en: https://rieoei.org/historico/deloslectores/1214Villarruel.pdf. Acceso en: 24 jul. 2019. 


\section{Juan Manuel Montero Peña}

Profesor titular de la Universidad de Moa, Cuba. Licenciado en Filosofía. Doctor en Ciencias Filosóficas. Máster en Ciencia, Tecnología, Sociedad. Coordinador de la "Maestría en desarrollo sustentable en la actividad minero - metalúrgica" de la Universidad de Moa "Dr. Antonio Núñez Jiménez", Cuba.

jmpena@ismm.edu.cu

jmmonteropena@gmail.com

\section{Hernando Hernández Pérez}

Profesor auxiliar de la Universidad de Moa "Dr. Antonio Núñez Jiménez", Cuba. Diplomado en Dirección. Profesor de Economía. Jefe de Departamento de Historia y Marxismo de la Universidad de Moa "Dr. Antonio Núñez Jiménez", Cuba.

hhernandez@ismm.edu.cu

\section{André Afonso Bambi}

Profesor asistente del Instituto Superior de Ciencias de Educación de Uige, Angola. Doctorando del Doctorado Curricular de Minería de la Universidad de Moa "Dr. Antonio Núñez Jiménez". Graduado de Ingeniero de Minas en el ISMM. Profesor de Geología General e histórica, de Topografía y de Ordenamiento Territorial.

andrebambi2@gmail.com 